\title{
Hand function and cost effectiveness after collagenase injection versus aponeurectomy for Dupuytren's contracture: results of the CeCORD-J study
}

Michiro Yamamoto ( $\sim$ michi-ya@med.nagoya-u.ac.jp )

Nagoya University

Hideo Yasunaga

Tokyo University

Ryosuke Kakinoki

Kindai University

Naoto Tsubokawa

Niigata Hand Surgery Foundation

Akimasa Morita

Suzuka Kaisei Hospital

Katsumi Tanaka

Nagasaki University

Akinori Sakai

the University of Occupational and Environmental Health

Toshikazu Kurahashi

Anjo Kosei Hospital

Hitoshi Hirata

Nagoya University

Masahiro Tatebe

Nagoya University

Tetsuro Onishi

Nagoya University

Katsuyuki Iwatsuki

Nagoya University

Katsuhiro Tokutake

Nagoya University

Kazuhiro Otani

Kindai University

Kunitaka Menuki

the University of Occupational and Environmental Health 


\section{Yoshiaki Yamanaka}

the University of Occupational and Environmental Health

\section{Shiro Urata}

Anjo Kosei Hospital

\section{Takeshi Oguchi}

Anjo Kosei Hospital

Norimasa Iwasaki

Hokkaido University

\section{Yuichiro Matsui}

Hokkaido University

Hiroyasu Ikegami

Toho University

\section{Hiroaki Sakano}

Hiratsuka Kyosai Hospital

\section{Tetsu Katsumura}

Hiratsuka Kyosai Hospital

\section{Masao Nishiwaki}

Kawasaki Municipal Hospital

\section{Toshikazu Tanaka}

Kikkoman General Hospital

\section{Yuichi Hirase}

Yotsuya Medical Cube

\section{Yuri Kanno}

Yotsuya Medical Cube

Hiroyuki Kato

Shinshu University

\section{Masanori Hayashi}

Shinshu University

\section{Shohei Omokawa}

Nara Medical University

\section{Hideo Hasegawa}

Nara Medical University

\section{Hiroyuki Gotani}

Osaka Ekisaikai Hospital

\section{Yoshitaka Tanaka}

Osaka Ekisaikai Hospital

\section{Toru Sunagawa}

Hiroshima University

\section{Rikuo Shinomiya}


Hiroshima University

\section{Rhoji Kajiwara}

Matsuyama Red Cross Hospital

\section{Etsuhiro Nakao}

Chunichi Hospital

\section{Takanobu Nishizuka}

Chunichi Hospital

\section{Yasunori Hattori}

Ogori Daiichi General Hospital

\section{Takaaki Shinohara}

Daido Hospital

\section{Kentaro Watanabe}

Nagoya Ekisaikai Hospital

Nobuyuki Okui

Yokkaichi Municipal Hospital

Hiroshi Koshima

Kani Tono Hospital

Tsuyoshi Tajika

Gunma University

\section{Hiroyuki Ohi}

Seirei Hamamatsu General Hospital

\section{Yoshio Kaji}

Kagawa University

\section{Eiichi Nagayoshi}

Asahi Kasei Pharma Corporation

\section{Ataru Igarashi}

Yokohama City University

\section{Research Article}

Keywords: Dupuytren's contracture, cost effectiveness, collagenase injection, aponeurectomy

Posted Date: June 25th, 2021

DOl: https://doi.org/10.21203/rs.3.rs-651200/v1

License: (c) (i) This work is licensed under a Creative Commons Attribution 4.0 International License. Read Full License 


\section{Abstract}

\section{Background}

This study compared hand function and the cost effectiveness of treatment between collagenase Clostridium histolyticum $(\mathrm{CCH})$ injection and limited fasciectomy for patients with Dupuytren's contracture (DC).

Materials and Methods

The CeCORD-J study is a prospective, multicenter, non-randomized controlled, observational study of two parallel groups. Participants were DC patients with multiple affected fingers, including flexion contracture of the proximal interphalangeal (PIP) joint. The primary outcome was the Hand10 score, as a patientreported outcome measure. We set secondary outcomes of EQ-5D-5L score, degree of extension deficit, and direct cost. Propensity score adjustment was used to balance differences in patient characteristics between groups.

Results

Participants comprised 52 patients in the Collagenase group and 26 patients in the Surgery group. Hand10 score was significantly better in the Collagenase group at 1 and 2 weeks. EQ-5D-5L score was significantly higher in the Collagenase group at 8 weeks. Mean direct cost was 248,000 yen higher in the Surgery group than in the Collagenase group. Extension deficit angle of the PIP joint was significantly larger in the Collagenase group at 26 weeks.

Conclusions

$\mathrm{CCH}$ injection provides better short-term hand function and cost effectiveness than surgery.

UMIN-CTR (UMIN000029826)

\section{Introduction}

Dupuytren's contracture (DC) is a fibroproliferative disease of the palmar hand. DC causes symptomatic and progressive flexion contractures of the digits ${ }^{1}$. Flexion contracture often occurs in the metacarpophalangeal (MP) and/or proximal interphalangeal (PIP) joints of the ring and little fingers ${ }^{2}$. Flexion contracture of the digits influences daily activities and quality of life (QOL) by limiting hand function ${ }^{3}$. DC has been considered common in Caucasians of Scandinavian and Celtic ancestry, but reports on DC from Asian and African countries have increased recently ${ }^{4,5}$. Both genetic and environmental factors are considered to affect disease progression, and the prevalence of DC increases with age ${ }^{6}$. 
Surgical procedures including fasciectomy, dermatofasciectomy, and needle fasciotomy have been standard treatment options for DC. Since Hurst et al. reported level 1 evidence for the utility of collagenase Clostridium histolyticum (CCH) injection compared with placebo in 2009, the United States Food and Drug Administration in 2010, the European Medicines Agency in 2011 and the Japanese Pharmaceuticals and Medical Devices Agency in 2015 have approved CCH injection for patients with $\mathrm{DC}^{5,7}$.

Hand function and degree of flexion contracture after treatment are the most important issues for patients with DC. Some reports have described upper limb function using patient-reported outcome measures (PROMs) such as Disabilities of the Arm, Shoulder and Hand (DASH) and the Michigan Hand Outcomes Questionnaire (MHQ) before and after surgical treatment for $\mathrm{DC}^{8,9,10}$. However, few papers have reported results from PROMs before and after $\mathrm{CCH}$ injection for patients with $\mathrm{DC}$. Furthermore, very few studies have prospectively compared results between surgery and $\mathrm{CCH}$ injection using PROMs.

Outcomes after treatment will differ depending on which joints are affected. Generally, flexion contracture of MP joints shows better prognosis than that of PIP joints. Treatment is more difficult for PIP joints than for MP joints because of the higher frequencies of recurrence and complications. A study of patients with DC-affected PIP joints is thus necessary.

The cost effectiveness of DC treatments has been reported from European and North American countries. Atroshi et al. reported costs for collagenase injections compared with fasciectomy in the treatment of DC as a retrospective cohort study in Sweden ${ }^{11}$. Although the follow-up period was as short as 6 weeks, treatment of $D C$ with one collagenase injection cost $33 \%$ less than fasciectomy, while offering equivalent efficacy of contracture reduction. Baltzer and Binhammer in Canada estimated that injectable collagenase would be feasible for treating DC affecting a single finger if it cost significantly less than the current United States pricing ${ }^{12}$. On the other hand, the National Health Service (NHS) in the United Kingdom simulated the costs of treatment for patients with DC affecting more than three joints, revealing limited fasciectomy as more cost effective than percutaneous needle fasciotomy or $\mathrm{CCH}$ injection. $\mathrm{CCH}$ injection showed the worst cost effectiveness for patients with DC affecting multiple fingers ${ }^{13}$. However, the costs of $\mathrm{CCH}$ injections, surgical procedures, and hand therapy differ between countries. Most patients are interested in the prices of treatment and often ask hand surgeons about the costs. Hand surgeons thus need cost and utility data as well as results for hand function after treatment for DC to provide patients with sufficient information on functional and economic aspects to make informed decisions before treatment.

The purpose of this study was to compare hand function and direct medical cost between $\mathrm{CCH}$ injection and limited fasciectomy for patients with DC-affected PIP joints. Our primary aim was to compare hand function between $\mathrm{CCH}$ injection and fasciectomy using the Hand10 PROM ${ }^{14}$. Secondary outcomes were set to compare QOL using the EQ-5D-5L ${ }^{15}$, patient satisfaction, degree of contracture, and direct cost. We hypothesized that no significant difference in hand function or QOL would be evident between groups, but that $\mathrm{CCH}$ injection would prove superior to fasciectomy in terms of cost effectiveness. 


\section{Materials And Methods}

The CeCORD-J study is a prospective, multicenter, non-randomized controlled, observational study with two parallel groups. This study provides the utmost respect for the treatment choices of patients.

\section{Study setting}

After the ethics committee approval of Nagoya University Hospital (2017-0506-4), the CeCORD-J study was approved by the academic research project committee of the Japanese Society for Surgery of the Hand (JSSH) as an official project in 2017. We invited participation from all major referral centers of hand surgery in Japan with at least one hand surgery specialist. A final total of 28 hand surgery centers accepted and joined this study. Patients were recruited from those centers. Investigators had to have specialist certification in hand surgery because $\mathrm{CCH}$ injection can only be performed by hand surgery specialists in Japan. All participating investigators had previously performed both $\mathrm{CCH}$ injection and fasciectomy before initiation of the study. Hand surgeons participating in this study held three meetings before starting this study to confirm the protocol.

\section{Ethics and dissemination}

Both treatment options are standard procedures for patients with DC in Japan. This study was not a randomized controlled study for intervention selection. We have the utmost respect for patient preferences in terms of treatment selection. This study was therefore not interventional but observational in nature. This policy offers substantial benefits for patient recruitment. Our major concern was bias in the number of cases in each group. One solution to this issue is propensity score adjustment after study completion.

\section{Information on the trial and patient approval}

Written information was used to explain the study to patients. Because this study was observational in nature, patients were able to select their preferred treatment after explanation of both treatments by hand surgery specialists. Hand surgery specialists obtained informed consent for participation from each patient after the decision-making process.

\section{Participants}

The inclusion criteria were: 1) patients with passive extension deficit in either only PIP joints or both PIP and MP joints; 2) flexion contractures in at least two fingers of one hand; 3 ) age $>20$ years; and 4) the ability to answer questionnaires in Japanese.

Exclusion criteria were: 1) recurrent contracture in the finger to be treated; 2) contraindications for $\mathrm{CCH}$ injection; 3) pregnancy or planning to become pregnant; 4) need for continued anticoagulation; 5) participation in another clinical study; 6 ) judgement that study participation would be inappropriate by the enrolling investigator. 


\section{Baseline assessment}

Baseline demographics of patients included age, sex, family history of DC, duration of symptoms, medical history, histories of smoking and drinking, involved fingers and joints, and PROMs for the hand (Hand10), EQ-5D-5L, and satisfaction (a 5-point scale).

\section{Interventions}

Patients requesting $\mathrm{CCH}$ injection were included in the Collagenase group. In this group, $0.58 \mathrm{mg}$ of $\mathrm{CCH}$ was injected into the cord of the affected finger. One day after injection, the affected finger was manipulated under local anesthesia or wrist block. Patients could receive additional $\mathrm{CCH}$ injections up to 3 times in total if necessary, at intervals of $>30$ days.

Patients hoping to undergo fasciectomy were included in the Surgery group. In this group, aponeurectomy was performed under general anesthesia or axillary block.

Patients in both groups received hand therapy including splinting based on the judgement of the surgeon. An attending hand surgery specialist decided whether inpatient or outpatient treatment was warranted. Generally, patients in the Surgery group required inpatient treatment, and those in the Collagenase group could be treated using either outpatient or inpatient treatment.

\section{Study outcomes}

Primary outcome

The primary outcome was the PROM score for the Hand10 before and 1, 2, 4, 8, and 26 weeks after intervention. The Hand10 is a valid instrument for patients with upper limb disorder, even in elderly individuals, as the questionnaire includes illustrations that provide favorable effects on reproducibility ${ }^{14}$. The Hand 10 questionnaire consists of 10 self-reported questions designed to measure upper extremity disability and symptoms. Scores for the Hand10 range from 0 to 100, with lower numbers indicating lower levels of disability.

\section{Secondary outcomes}

We set the following secondary outcomes: 1) EQ-5D-5L (QOL) score; 2) patient satisfaction; 3 ) degrees of extension deficit and flexion of the treated joint, and 4) direct medical costs. We also investigated: 1) complications; and 2) recurrences as other outcomes. Recurrences were assessed 1 year after treatment, and all other secondary outcomes were evaluated up to 26 weeks after interventions.

Recurrence was considered to have occurred if the degree of extension deficit worsened by more than $30^{\circ}$ after treatment. We classified complications as: grade I, minor complications without needing any unexpected surgery or anesthesia; or grade II, major complications needing unexpected surgery under anesthesia or inpatient treatment. Each outcome was compared with baseline data. 


\section{Discontinuation criteria}

Discontinuation criteria were: 1 ) when the patient requested to withdraw from the study; 2) when the patient was found not to satisfy eligibility criteria after registration; 3) when continuation of the study was difficult because symptoms or complications worsened; or 4) when the hand surgery specialist decided on discontinuation for any other reason. When discontinuation occurred, data collection was stopped, but data collected up to the time of discontinuation were retained for use in the study.

\section{Power analysis}

Setting values of $a=0.05$ and $\beta=0.8$, sample size " $n$ " was calculated as $n=16 s^{2} / d^{2}$, where "s" is the standard deviation and " $\mathrm{d}$ " is the difference between two groups. According to previous papers, mean DASH score at 1 year postoperatively was 12.7 (standard deviation, 3.6$)^{8}$, and mean DASH score at 1 year after $\mathrm{CCH}$ injection was 7 (standard deviation, 9) ${ }^{16}$. We did not use DASH score, but Hand 10 was expected to show similar results. We therefore used "s" as 9, and "d" as 5.7 for sample size calculations. If both groups were to include the same number of patients, a sample size of 40 per group was required.

\section{Statistical analysis}

We compared baseline patient demographics between groups using the unpaired t-test or chi-square test. Baseline patient demographics included age, sex, number of involved fingers and joints, degree of extension deficit, Hand10 score, EQ-5D-5L (QOL) score, and satisfaction.

As the primary outcome, Hand10 scores were compared between groups using the unpaired t-test.

EQ-5D-5L (QOL) score, satisfaction, and degree of extension deficit were compared between groups as secondary outcomes using the unpaired t-test. Satisfaction was compared between groups using the Mann-Whitney $U$ test. We compared mean direct medical costs between groups.

Prevalence and severity of complications were summarized and compared between groups using the chisquare test.

Propensity score adjustment was used to balance differences in patient characteristics between Collagenase and Surgery groups. In a logistic regression model with treatment assignment variables (Collagenase group $=0$, Surgery group $=1$ ) as the dependent variables, the following independent variables were introduced to obtain the propensity score (= probability of undergoing surgery) for each patient: age, sex, total number of affected joints per patient, total number of affected fingers per patient, family history of DC, duration of symptoms, smoking, drinking, past or present history of diabetes mellitus, epilepsy, history of hand trauma, malignant tumor, baseline Hand10 score, baseline degree of extension defect of the primary PIP joint, degree of extension of the primary MP joint, and baseline EQ-5D$5 \mathrm{~L}(\mathrm{QOL})$ score. 
Outcomes were then compared between treatment groups after adjusting for propensity scores.

Specifically, a generalized linear model with treatment assignment variables (Collagenase group $=0$;

Surgery group $=1$ ) and propensity scores as independent variables and outcomes as dependent variables was used to determine differences in outcomes and associated $95 \%$ confidence intervals and p-values in the collagenase-controlled Surgery group.

The outcome variables examined were: Hand10 score (weeks 1, 2, 4, 8, and 26), EQ-5D-5L (QOL) score (weeks 1, 2, 4, 8, and 26), direct cost, degree of extension deficit of the PIP (weeks 4 and 26), degree of extension deficit of the MP (weeks 4 and 26), degree of PIP flexion (weeks 4 and 26), and degree of MP flexion (weeks 4 and 26).

Statistical analysis was performed using STATA version 16 software.

\section{Data management}

We used a central monitoring system in the data center. Each hand surgery center registered anonymized patient information to the data center. Hand surgery specialists noted patient data at each time point and those data were stored at each hand surgery center. Anonymized patient data were collected at the data center by mail after finishing the follow-up period. This study was monitored by an independent assessor during patient recruitment and after completion of the study.

This study followed and respected the Declaration of Helsinki and the principles of good clinical practice.

\section{Results}

\section{Participants}

A total of 98 participants were initially enrolled in this study from April 2018 to July 2019. Of those, 20 were excluded, and data from 52 patients in the Collagenase group and 26 patients in the Surgery group (total cohort, 78 patients) were analyzed. The 20 patients were excluded due to contracture in only 1 finger $(n=10)$, dropout $(n=9)$, or voluntary withdrawal from the study before treatment $(n=1)$ (Figure 1$)$. Participant demographics are shown in Table 1. In the Collagenase group, 5 of 52 patients received a second injection at different sites.

\section{Primary outcome}

The time course for Hand10 score as primary outcome is shown in Figure 2. Hand10 scores were significantly higher in the Surgery group than in the Collagenase group at 1 and 2 weeks after treatment.

\section{Secondary outcomes}

The outcomes of EQ-5D-5L (QOL) score, direct cost, recurrence, complications, and satisfaction are summarized in Table 2. EQ-5D-5L (QOL) score was significantly higher in the Collagenase group than in the Surgery group at 2 weeks after treatment (Figure 3). The direct cost for the Collagenase group was 
370,000 yen (about $\$ 3395$ US dollars (USD) at an exchange rate of $\$ 1=109$ yen) on average, compared to 580,000 yen (about $\$ 5321$ ) for the Surgery group, representing a significant difference.

Recurrences were more frequent in the Collagenase group than in the Surgery group (18\% vs $9.5 \%$ ).

All complications in both groups were minor without additional surgery or anesthesia.

No significant difference in satisfaction was seen between groups. All patients in the Surgery group provided evaluations above neutral.

As other evaluation items, the time courses of extension deficit angle and flexion angle of the PIP and MP joints are shown in Table 3. No significant difference in extension deficit angle of the PIP or MP joint was seen between groups (Figure 4). Flexion angles of the PIP joint at week 4 and of the MP joint at weeks 4 and 26 were significantly smaller in the Surgery group than in the Collagenase group (Figure 5).

\section{Analysis with propensity score adjustment}

Results from propensity score adjustment are presented in Table 4. Hand10 score was significantly higher in the Surgery group at 1 and 2 weeks, but not at other time points. No significant difference in EQ$5 \mathrm{D}-5 \mathrm{~L}$ (QOL) score was seen after adjusting for propensity scores, except at 8 weeks. The direct cost was 248,000 yen (about $\$ 2275$ ) higher in the Surgery group than in the Collagenase group on average. A significant difference in extension deficit angle of the PIP joint was apparent between groups at 26 weeks. Although flexion angle after intervention was smaller in the Surgery group, no significant difference was evident between groups after propensity score adjustment.

\section{Discussion}

This multicenter, prospective observational study compared outcomes between $\mathrm{CCH}$ injection and aponeurectomy. This study was unique in that we included only patients with multiple affected fingers, including flexion contracture of the PIP joint, which is difficult to treat. This study did not randomize patients, and treatment groups were divided according to the preferences of the patient. The Collagenase group was a double of the Surgery group, because many patients preferred treatment with $\mathrm{CCH}$ injection.

Change in Hand10 score, as the primary outcome, showed a significant difference between the acute phase of weeks 1 and 2 after treatment, indicating that patients injected with $\mathrm{CCH}$ were able to return to daily life earlier than patients who underwent surgery. The results of propensity score adjustment to balance differences in patient backgrounds between groups also revealed significant differences in Hand10 score at 1 and 2 weeks. Zhou et al. compared $\mathrm{CCH}$ injection with limited fasciectomy using propensity score matching and showed significant improvements in activities of daily living, work performance, and satisfaction in their collagenase group compared to surgery group using the Michigan Hand Outcomes Questionnaire ${ }^{9}$. In the present study, the Collagenase group showed better PROMs than the Surgery group in the short-term, similarly as reported by Zhou et al. 
EQ-5D-5L (QOL) score was significantly higher in the Collagenase group than in the Surgery group after 2 weeks. However, after propensity score adjustment, a significant difference was only observed at 8 weeks. Given the difference of about 248,000 yen (about $\$ 2275$ ) in direct costs even if the EQ-5D-5L (QOL) score is almost equivalent between groups, collagenase appears to offer better short-term costeffectiveness by 26 weeks, even for those patients with multiple affected joints, including PIP joints. The price of collagenase in Japan is 197,202 yen, and 24,900 yen is added as a procedure fee, for a total of 222,102 yen (about $\$ 2038$ ). On the other hand, the cost of surgery is 224,800 yen for two or three affected fingers, plus 1,700 yen for regional anesthesia, for a total of 226,500 yen (about \$2078). No significant difference in cost was seen between collagenase injection and surgery itself, but hospitalization and rehabilitation visits led to a difference in total direct costs. $\mathrm{CCH}$ injection is feasible for treating DC affecting a single finger, according to a Canadian cost-utility analysis ${ }^{12}$. Conversely, the $\mathrm{NHS}$ has estimated that $\mathrm{CCH}$ injection would yield the worst cost effectiveness for DC involving more than three joint contractures ${ }^{13}$. Although the follow-up period was short, at 26 weeks, the present study provided evidence for the cost effectiveness of $\mathrm{CCH}$ injection even for hands affected in multiple fingers, including PIP joints.

No significant difference in satisfaction was seen between groups using the 5-point scale, and all patients in the Surgery group provided evaluations above neutral. The satisfaction of patients in both groups was not poor, regardless of cost-effectiveness.

The number of complications tended to be higher in the Collagenase group. Skin laceration was the most frequent complication in the Collagenase group, and no serious complications showing a causal relationship to the treatment were encountered. Recurrence also tended to be more frequent in the Collagenase group. Recurrences were reported in 160 of 199 digits (80\%) at an average of 7.2 years after collagenase injection despite a heterogeneous patient population ${ }^{17}$. Furthermore, Yoon et al. simulated recurrent DC in a 60-year-old patient and concluded that collagenase injections are not a cost-effective intervention and should not be preferred over percutaneous needle aponeurotomy or limited fasciectomy ${ }^{18}$. Leafblad et al. retrospectively reviewed 848 interventions for DC, and reported 2-year reintervention rates following needle aponeurotomy, collagenase, and fasciectomy of $24 \%, 41 \%$, and $4 \%$, respectively, and 5 -year rates of $61 \%, 55 \%$, and $4 \%$, respectively ${ }^{19}$. Cumulative costs for possible reinterventions thus also need to be taken into consideration.

A significant difference in finger flexion angle was seen between groups after treatment. Flexion angles of both the PIP and MP joints were reduced in the Surgery group. This phenomenon often occurs clinically, and although finger extension improves after surgery, bending the joint deeply when grasping becomes difficult. This effect is attributed to swelling of the fingers, surgical scarring, and tendon adhesion. Rehabilitation measures such as passive range-of-motion training were required after surgery. In the Collagenase group, the decrease in flexion angle was slight and rehabilitation was often not required, which was considered to affect the cost. However, after adjusting for propensity scores, no significant difference in finger flexion angle was evident between groups. 
This study provided comprehensive evidence for a comparison of collagenase injection and surgery for DC with contractures of more than two fingers including a PIP joint. However, some limitations must be kept in mind when interpreting the findings from this study. First, some degree of selection bias would be present, because this was a prospective observational study and patients were not randomly assigned. Surprisingly, the two groups showed no significant differences in background characteristics other than the number of patients. Furthermore, propensity score adjustments were performed to balance differences in patient background between groups without reducing the number of participants eligible for analysis. Second, recurrence was evaluated at 52 weeks, while all other outcomes had a follow-up period of 26 weeks. Although the financial issue of multi-institutional, long-term follow-up study needs to be considered, comparison of longer-term results is certainly desirable. However, for the elderly individuals who represent the majority of patients with this disease, short-term results are also important.

\section{Conclusion}

For DC with multiple affected fingers, including flexion contracture of the PIP joint, this multicenter, prospective, observational study was conducted by dividing patients into an injectable collagenase group and a surgical group, giving priority to the wishes of the patient. Propensity scores were adjusted to balance differences in patient background between groups. As a primary outcome, the PROM of Hand10 score was significantly better in the Collagenase group at 1 and 2 weeks after treatment, but no differences were evident between groups at 26 weeks. EQ-5D-5L (QOL) score tended to be higher in the Collagenase group, and the direct cost was 248,000 yen (about $\$ 2275$ US dollars at the exchange rate of $\$ 1=109$ yen) higher in the Surgery group than in the Collagenase group. Short-term cost-effectiveness appeared better in the Collagenase group. The degree of extension deficit of the PIP joint after treatment was significantly larger in the Collagenase group, and minor complications and recurrences tended to be more frequent in the Collagenase group. The CeCORD-J study was able to compare comprehensive data on hand function and cost effectiveness between collagenase injection and surgery. The results of this study will help in treatment decisions.

\section{Declarations}

\section{Acknowledgement:}

Research reported in this publication was supported by the Japanese Society for Surgery of the Hand as an academic research project in 2017.

\section{Competing interest:}

The authors disclosed receipt of the following financial support for the research, authorship, and/or publication of this article. This study was supported by Asahi Kasei Pharma Corporation. However, research planning, implementation, and analysis were carried out by multiple collaborators, and the research results were not manipulated so as to arbitrarily lead to favorable results for the company. 


\section{Author contributions Statement:}

Yamamoto, Yasunaga, and Hirata had full access to all of the data in the study and take responsibility for the integrity of the data and the accuracy of the data analysis.

Study concept and design: Hirata, Yasunaga, Igarashi, Tanaka, Kakinoki, Sakai, Ikegami, and Yamamoto.

Acquisition of data: Yamamoto, Kakinoki, Tsubokawa, Morita, Tanaka, Sakai, Kurahashi, and Hirata.

Analysis and interpretation of data: Yamamoto and Yasunaga.

Drafting of the manuscript: Yamamoto, Yasunaga, Nagayoshi, and Hirata.

Critical revision of the manuscript for important intellectual content: Yamamoto, Yasunaga, Nagayoshi, and Hirata.

Statistical analysis: Yamamoto and Yasunaga.

Administrative, technical, or material support: Nagayoshi, Yasunaga, and Hirata.

Study supervision: Yamamoto, Yasunaga, and Hirata.

Preparation of figures 1-5: Yamamoto.

All authors reviewed the manuscript.

\section{References}

1. Shih B, Bayat A. Scientific understanding and clinical management of Dupuytren disease. Nat Rev Rheumatol. 2010;6:715-726.

2. Dias JJ, Singh HP, Ullah A, Bhowal B, \& Thompson JR. Patterns of recontracture after surgical correction of Dupuytren disease. J Hand Surg Am. 2013;38:1987-1993.

3. Engstrand C, Krevers B, Nylander G, \& Kvist J. Hand function and quality of life before and after fasciectomy for Dupuytren contracture. J Hand Surg Am. 2014;39:1333-1343.

4. Gebereegziabher A, Baraki A, Kebede Y, Mohammed I, \& Finsen V.

Dupuytren's contracture in Ethiopia. J Hand Surg Eur. 2017;42:26-28.

5. Hirata $\mathrm{H}$, et al. Efficacy and safety of collagenase Clostridium histolyticum injection for Dupuytren's contracture in non-Caucasian Japanese patients (CORD-J Study): the first clinical trial in a non-Caucasian population. J Hand Surg Eur. 2017;42:30-38. 
6. Lanting R, Broekstra DC, Werker PM, \& van den Heuvel ER. A systematic review and meta-analysis on the prevalence of Dupuytren disease in the general population of Western countries. Plast Reconstr Surg. 2014;133:593-603.

7. Hurst LC, et al. Injectable collagenase clostridium histolyticum for Dupuytren's contracture. N Engl J Med. 2009;361:968-979.

8. Rodrigues $\mathrm{J}$, et al. Recovery, responsiveness and interpretability of patient-reported outcome measures after surgery for Dupuytren's disease. J Hand Surg Eur. 2017;42:301-309.

9. Zhou C, et al. Collagenase clostridium histolyticum versus limited fasciectomy for Dupuytren's contracture: outcomes from a multicenter propensity score matched study. Plast Reconstr Surg. 2015;136:87-97.

10. Beaudreuil J, et al. Unité Rhumatologique des Affections de la Main (URAM) scale: Development and validation of a tool to assess Dupuytren's disease-specific disability. Arthritis Care Res (Hoboken). 2011;63:1448-1455.

11. Atroshi I, Strandberg E, Lauritzson A, Ahlgren E, \& Waldén M. Costs for collagenase injections compared with fasciectomy in the treatment of Dupuytren's contracture: a retrospective cohort study. BMJ open. 2014;4:e004166.

12. Baltzer $\mathrm{H}$, \& Binhammer PA. Cost-effectiveness in the management of Dupuytren's contracture. A Canadian cost-utility analysis of current and future management strategies. Bone Joint J. 2013;95:10941100.

13. Brazzelli $\mathrm{M}$, et al. Collagenase clostridium histolyticum for the treatment of dupuytren's contracture: systematic review and economic evaluation. Health Technol Assess. 2015;19:1-202.

14. Kurimoto S, et al. Development and validation of a ten-item questionnaire with explanatory illustrations to assess upper extremity disorders: favorable effect of illustrations in the item reduction process. J Orthop Sci. 2011;16:737-744.

15. Shiroiwa T, et al. Japanese population norms for preference-based measures: EQ-5D-3L, EQ-5D-5L, and SF-6D. Qual Life Res. 2016;25:707-719.

16. Leclère F-M, Mathys $L, \&$ Vögelin E. Collagenase injection in Dupuytren's disease, evaluation of the ultrasound assisted technique. Chir Main. 2014;33:196-203.

17. Zhang D, Earp BE, Benavent KA, \& Blazar P. Collagenase Treatment of Dupuytren's Disease with Minimum 5-Year Follow-Up: Recurrence, Reintervention, and Satisfaction. Plast Reconstr Surg. 2020;146:1071-1079. 
18. Yoon AP, Kane RL, Hutton DW, \& Chung KC. Cost-effectiveness of Recurrent Dupuytren Contracture Treatment. JAMA Netw Open. 2020;3:e2019861.

19. Leafblad ND, et al. Outcomes and Direct Costs of Needle Aponeurotomy, Collagenase Injection, and Fasciectomy in the Treatment of Dupuytren Contracture. J Hand Surg Am. 2019;44:919-927.

\section{Tables}

Table 1. Demographic and baseline clinical characteristics of participants

\begin{tabular}{|c|c|c|c|}
\hline Group & Collagenase & Surgery & $\begin{array}{l}P \\
\text { value }\end{array}$ \\
\hline Number of participants & 52 & 26 & \\
\hline Age, years (mean \pm SD) & $71 \pm 8.9$ & $70 \pm 7.6$ & 0.63 \\
\hline \multirow[t]{2}{*}{ Sex } & Male: 50 & Male: 25 & 1 \\
\hline & Female: 2 & Female: 1 & \\
\hline Total affected joint per patient (mean \pm SD) & $3.8 \pm 1$ & $4.2 \pm 0.9$ & 0.15 \\
\hline Total affected finger per patient (mean \pm SD) & $2.2 \pm 0.5$ & $2.2 \pm 0.4$ & 1 \\
\hline Family history of DC, n (\%) & $5(10)$ & $1(4)$ & 0.3 \\
\hline Duration of symptoms, month (mean \pm SD) & $14 \pm 32.7$ & $\begin{array}{l}24.3 \pm \\
35.6\end{array}$ & 0.31 \\
\hline \multicolumn{4}{|l|}{ History of risk factors } \\
\hline Smoking, n (\%) & $15(29)$ & $4(15)$ & 0.19 \\
\hline Drinking, n (\%) & $40(77)$ & $21(81)$ & 0.78 \\
\hline Diabetes mellitus, n (\%) & $12(23)$ & $11(42)$ & 0.08 \\
\hline Epilepsy, n (\%) & $1(2)$ & $1(4)$ & 0.61 \\
\hline History of hand trauma, n (\%) & $3(6)$ & $1(4)$ & 0.71 \\
\hline Malignant tumor, n (\%) & $9(17)$ & $3(12)$ & 0.51 \\
\hline Hand10 score (mean \pm SD) & $15.5 \pm 18.2$ & $\begin{array}{l}14.2 \pm \\
15.4\end{array}$ & 0.76 \\
\hline $\begin{array}{l}\text { Degree of extension deficit of the primary PIP joint (mean } \pm \\
\text { SD) }\end{array}$ & $37.2 \pm 23.6$ & $\begin{array}{l}46.9 \pm \\
27.8\end{array}$ & 0.11 \\
\hline $\begin{array}{l}\text { Degree of extension deficit of the primary MP joint (mean } \pm \\
\text { SD) }\end{array}$ & $39.9 \pm 20.5$ & $\begin{array}{l}36.5 \pm \\
21.9\end{array}$ & 0.5 \\
\hline
\end{tabular}


DC, Dupuytren's contracture; MP, metacarpophalangeal; PIP, proximal interphalangeal; SD, standard deviation.; n, number

Table 2. Comparison of secondary outcomes

\begin{tabular}{llll} 
Group & Collagenase & Surgery & P value \\
\hline EQ-5D-5L score & & & \\
Baseline (mean \pm SD) & $0.859 \pm 0.145$ & $0.81 \pm 0.121$ & 0.14 \\
$26 \mathrm{w}$ follow up (mean \pm SD) & $0.905 \pm 0.134$ & $0.841 \pm 0.119$ & $<0.05$ \\
\hline Direct cost, yen (mean \pm SD) & $372370 \pm 262517$ & $582147 \pm 267204$ & $<0.01$ \\
\hline Recurrence, $\mathrm{n}(\%)$ & $8(18)$ & $2(9.5)$ & 0.38 \\
\hline Complication, $\mathrm{n}(\%)$ & $9(17)$ & $2(8)$ & 0.25 \\
\hline Injection site laceration, $\mathrm{n}(\%)$ & $5(10)$ & & \\
Numbness of the finger, $\mathrm{n}(\%)$ & $1(2)$ & $1(4)$ & \\
Injection site blister, $\mathrm{n}(\%)$ & $1(2)$ & & \\
Arthritis of the finger, $\mathrm{n}(\%)$ & $1(2)$ & & \\
Pneumonia, $\mathrm{n}(\%)$ & $1(2)$ & $1(4)$ & \\
hypertrophic scar, $\mathrm{n}(\%)$ & & & \\
\hline Satisfaction \#\# & & 12 & \\
1 very satisfied & & 9 & \\
2 satisfied & 18 & 5 & \\
3 neutral & 24 & 0 & \\
4 dissatisfied & 4 & & \\
5 very dissatisfied & 3 & & \\
\hline
\end{tabular}

SD, standard deviation.

\#: The number of patients who were evaluated at 52 weeks in Collagenase and Surgery group was 45 and 21 , respectively.

\#\#: Data of 3 patients in collagenase group was absent.

Table 3. Time course of Hand10 score, degree of contracture, and flexion angle 


\begin{tabular}{|c|c|c|c|}
\hline Group & Collagenase & Surgery & $P$ value \\
\hline \multicolumn{4}{|l|}{ Hand10 score } \\
\hline Baseline (mean \pm SD) & $15.5 \pm 18.2$ & $14.2 \pm 15.4$ & 0.76 \\
\hline $1 \mathrm{w}($ mean $\pm \mathrm{SD})$ & $25.2 \pm 23.7$ & $46.1 \pm 23.8$ & $<0.01$ \\
\hline $2 \mathrm{w}($ mean $\pm S D)$ & $18.2 \pm 20.6$ & $36.2 \pm 25$ & $<0.01$ \\
\hline $4 \mathrm{w}($ mean $\pm S D)$ & $10.2 \pm 13.9$ & $16.8 \pm 16.8$ & 0.07 \\
\hline $8 \mathrm{w}($ mean $\pm \mathrm{SD})$ & $8.1 \pm 12.8$ & $12.7 \pm 14.5$ & 0.15 \\
\hline $26 \mathrm{w}($ mean $\pm S D)$ & $6.5 \pm 10.3$ & $7.9 \pm 11.3$ & 0.59 \\
\hline \multicolumn{4}{|c|}{ PIP degree of extension deficit } \\
\hline Baseline (mean \pm SD) & $37.2 \pm 23.6$ & $46.9 \pm 27.8$ & 0.11 \\
\hline $4 \mathrm{w}($ mean $\pm \mathrm{SD})$ & $17.2 \pm 19.4$ & $12.8 \pm 14.9$ & 0.32 \\
\hline $26 \mathrm{w}($ mean $\pm S D)$ & $20.6 \pm 21.1$ & $14.6 \pm 15.6$ & 0.21 \\
\hline \multicolumn{4}{|c|}{ MP degree of extension deficit } \\
\hline Baseline (mean \pm SD) & $39.9 \pm 20.5$ & $36.5 \pm 21.9$ & 0.5 \\
\hline $4 \mathrm{w}($ mean $\pm S D)$ & $7.3 \pm 12.8$ & $3.7 \pm 6.4$ & 0.18 \\
\hline 26w $($ mean $\pm S D)$ & $4.9 \pm 9$ & $4.5 \pm 9.8$ & 0.86 \\
\hline \multicolumn{4}{|l|}{ PIP degree of flexion } \\
\hline Baseline (mean \pm SD) & $93.8 \pm 5.3$ & $93.7 \pm 7.7$ & 0.92 \\
\hline $4 \mathrm{w}($ mean $\pm \mathrm{SD})$ & $91.6 \pm 7.9$ & $86.6 \pm 10.4$ & $<0.05$ \\
\hline $26 \mathrm{w}($ mean $\pm S D)$ & $94.2 \pm 7.1$ & $93.5 \pm 6.1$ & 0.68 \\
\hline \multicolumn{4}{|l|}{ MP degree of flexion } \\
\hline Baseline (mean \pm SD) & $85.9 \pm 6.8$ & $84.6 \pm 6.3$ & 0.43 \\
\hline $4 \mathrm{w}($ mean $\pm S D)$ & $84.1 \pm 10.9$ & $79.1 \pm 8.6$ & $<0.05$ \\
\hline $26 \mathrm{w}($ mean $\pm S D)$ & $87.5 \pm 8.1$ & $82.6 \pm 6$ & $<0.01$ \\
\hline
\end{tabular}

w, week; MP, metacarpophalangeal; PIP, proximal interphalangeal; SD, standard deviation.

Table 4. Results of propensity-score adjusted generalized linear models for comparing outcomes between Collagenase and Surgery groups 
Coefficient

Hand10 score

\begin{tabular}{llllll}
\hline $1 w$ & 13.6 & 0.0 & to & 27.1 & 0.05 \\
\hline $2 w$ & 14.3 & 2.0 & to & 26.6 & $<0.05$ \\
\hline $4 w$ & 3.0 & -5.8 & to & 11.8 & 0.51 \\
\hline $8 w$ & 2.7 & -4.8 & to & 10.2 & 0.48 \\
\hline $26 w$ & 0.9 & -5.2 & to & 7.0 & 0.77
\end{tabular}

EQ-5D-5L score

\begin{tabular}{llllll}
\hline $1 w$ & -0.030 & -0.092 & to 0.031 & 0.34 \\
\hline $2 w$ & -0.055 & -0.116 & to 0.006 & 0.08 \\
\hline $4 w$ & -0.035 & -0.100 & to & 0.029 & 0.28 \\
\hline $8 w$ & -0.090 & -0.154 & to & -0.027 & $<0.01$ \\
\hline $26 w$ & -0.066 & -0.139 & to & 0.007 & 0.08 \\
\hline Direct cost, yen & 248,375 & 104,249 & to & 392,501 & $<0.01$ \\
\hline PIP degree of extension deficit & & & & & \\
\hline $4 w$ & -12.0 & -21.6 & to & -2.5 & 0.01 \\
\hline $26 w$ & -14.5 & -25.1 & to -3.8 & $<0.01$ \\
\hline MP degree of extension deficit & & & & & \\
\hline $4 w$ & -5.7 & -13.8 & to 2.4 & 0.17 \\
\hline $26 w$ & -0.7 & -8.2 & to 6.7 & 0.85
\end{tabular}

PIP degree of flexion

\begin{tabular}{llllll}
\hline $4 \mathrm{w}$ & -4.1 & -9.0 & to 0.8 & 0.1 \\
\hline $26 \mathrm{w}$ & -1.5 & -5.2 & to 2.2 & 0.42 \\
\hline MP degree of flexion & & & & & \\
\hline $4 \mathrm{w}$ & -3.9 & -9.5 & to & 1.7 & 0.17 \\
\hline $26 \mathrm{w}$ & -3.1 & -7.1 & to & 0.9 & 0.13 \\
\hline
\end{tabular}

Coefficients (and their 95\% confidence intervals) indicate the degree of each outcome in the Surgery group with reference to the Collagenase group.

w, week

Page $18 / 21$ 


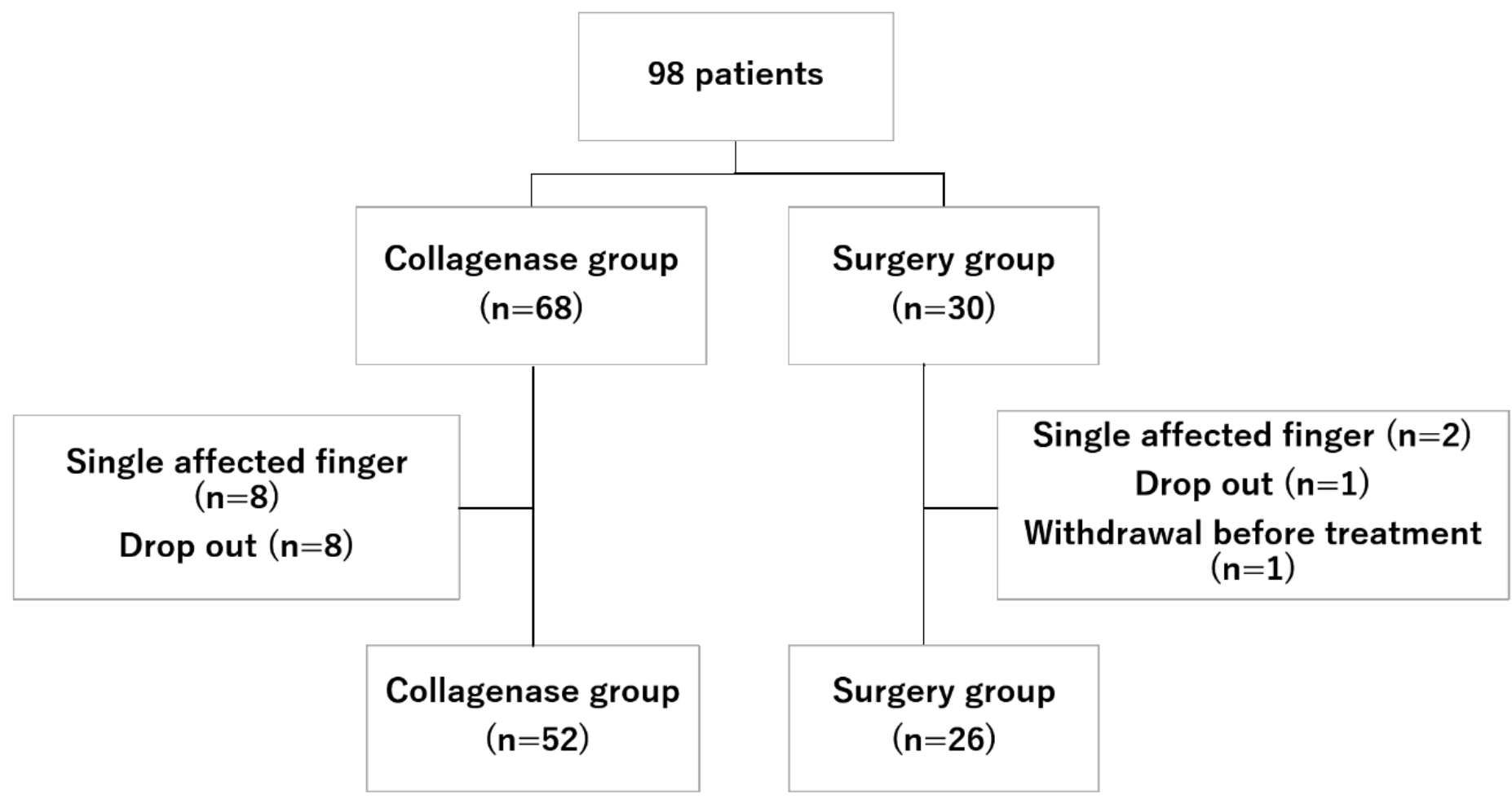

Figure 1

Study flowchart Twenty patients were excluded because of single affected finger $(n=10)$, loss to follow-up $(n=9)$, or withdrawal before treatment $(n=1) \cdot n$, number

\section{Hand10 score}

80

60

40

20

0

$-20$

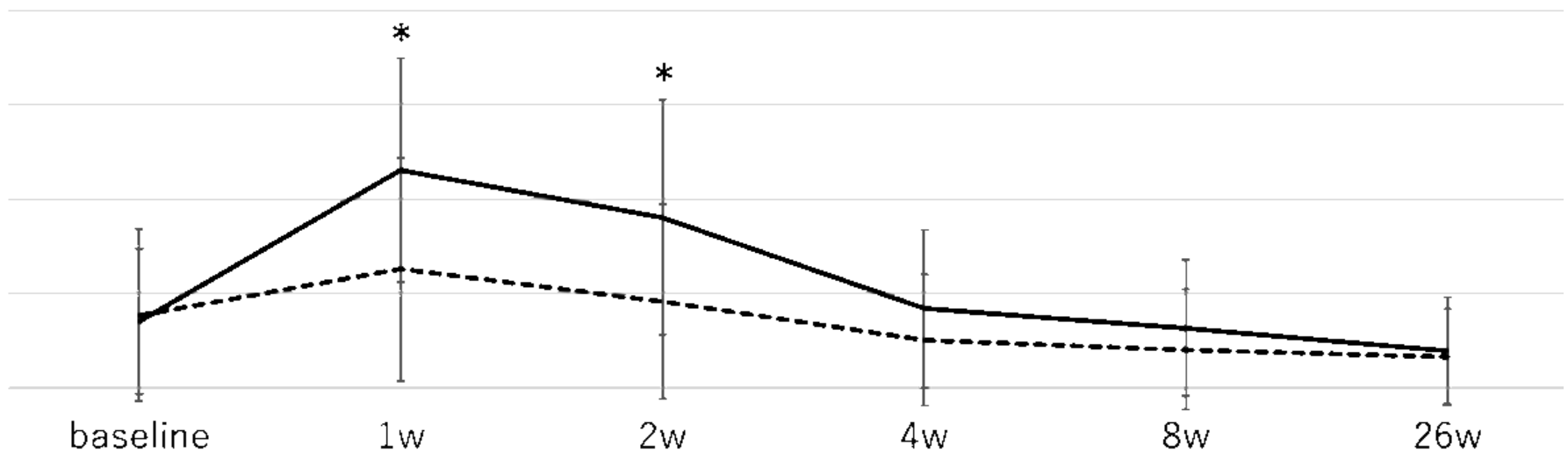

$$
\text { --- collagenase — surgery }
$$$$
*: p<0.01
$$

Figure 2 
Time course for Hand10 scores Hand10 score was assessed as the primary outcome. Hand10 scores were significantly higher in the collagenase group compared to the surgery group at both 1 week and 2 weeks. w, week

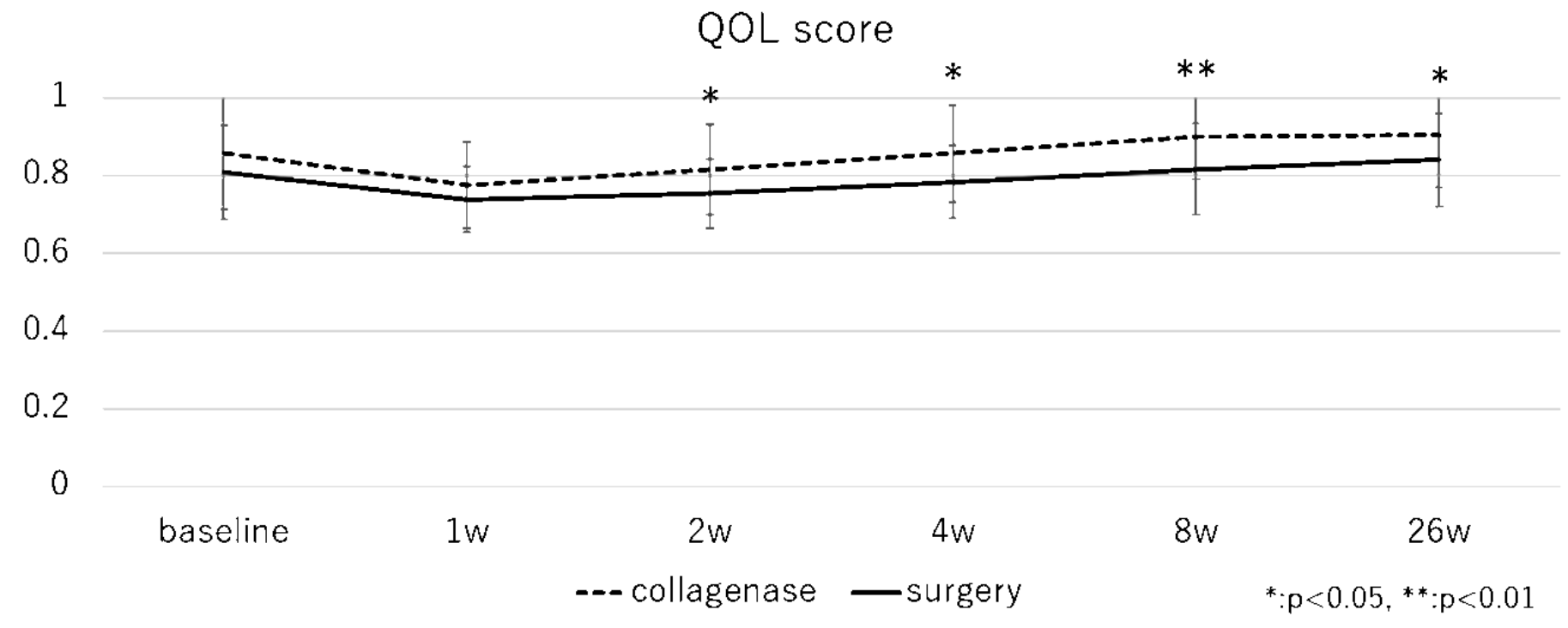

Figure 3

Time course for EQ-5D-5L (QOL) scores QOL scores were significantly higher in the Collagenase group than in the Surgery group from 2 weeks to 26 weeks. w, week

PIP degree of extension deficit

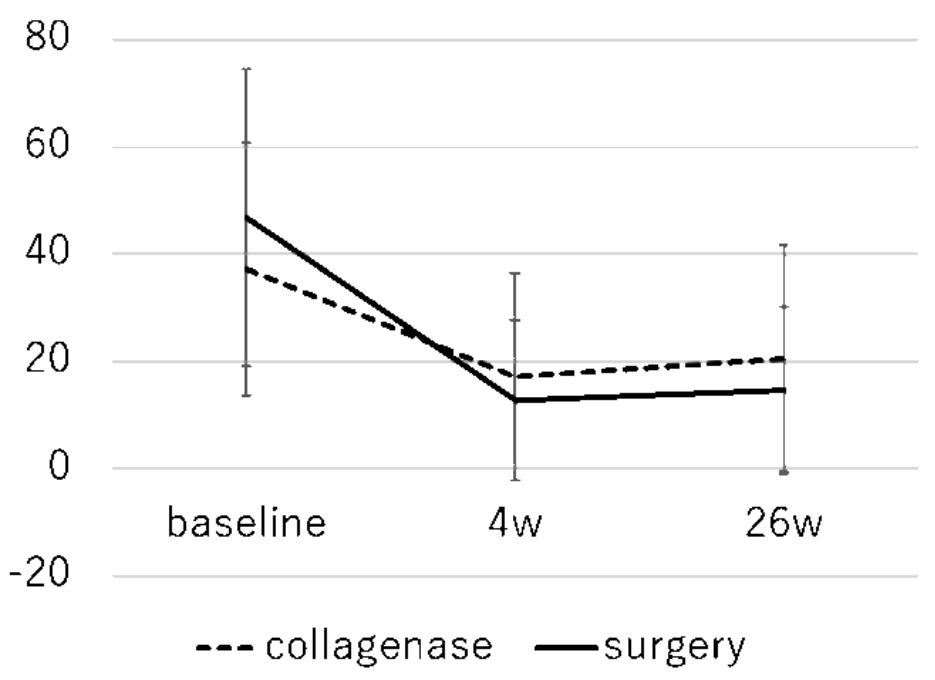

MP degree of extension deficit

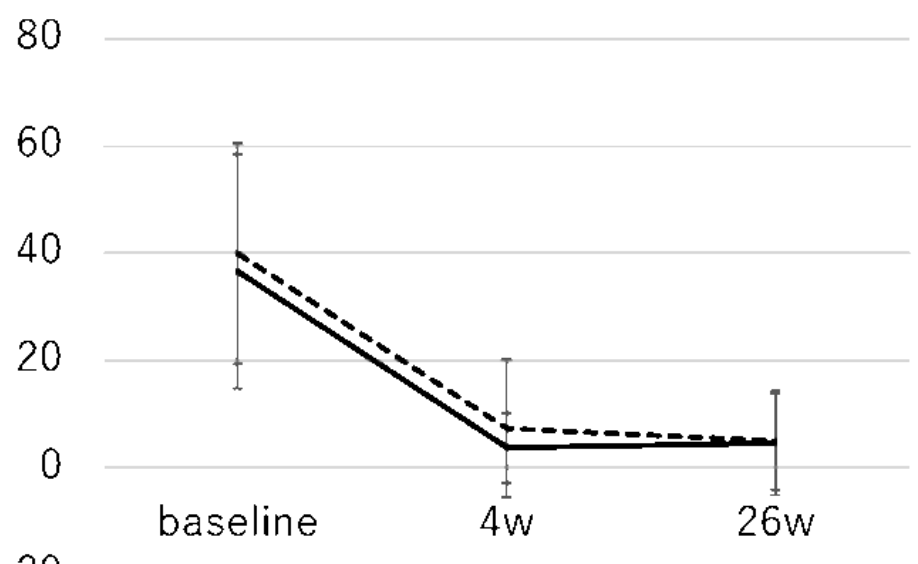
$-20$

Figure 4

Time course for degree of contracture No significant difference was identified between groups. w, week 
PIP degree of flexion

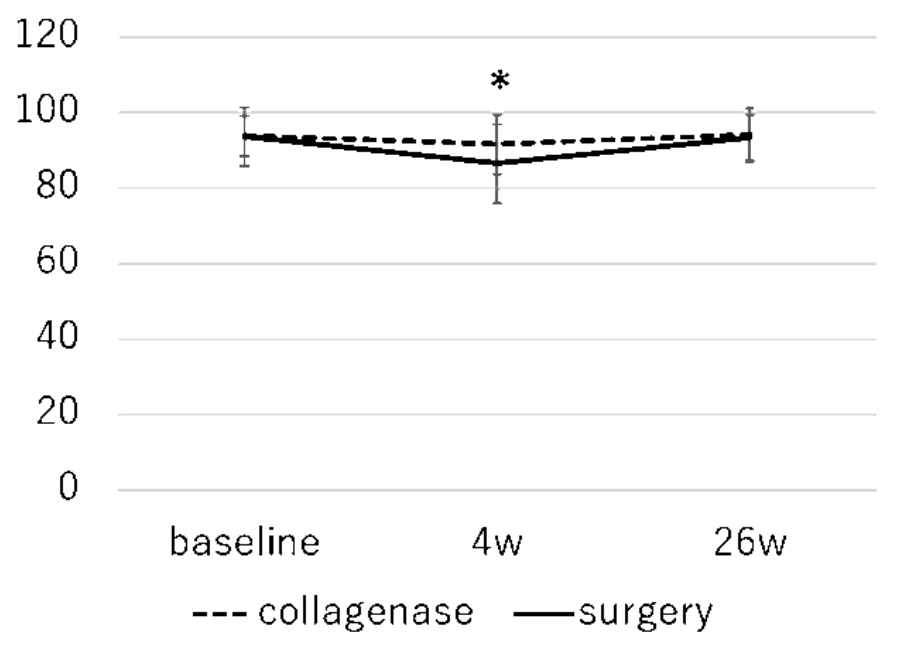

MP degree of flexion

120

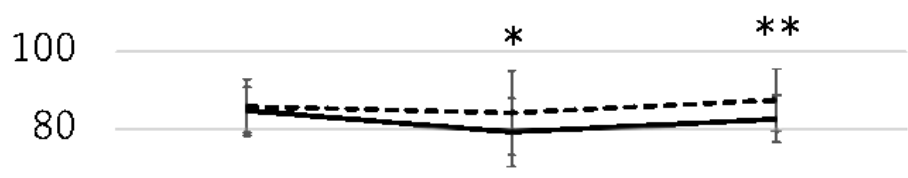

60

40

20

0

$$
\begin{aligned}
& \text { baseline } 4 w \quad 26 w \\
& \text {--- collagenase -surgery } \quad{ }^{*}: p<0.05 \text {, }
\end{aligned}
$$

\section{Figure 5}

Time course for flexion angle Mean flexion angle of the PIP joint was significantly smaller in the Surgery group than in the Collagenase group at 4 weeks. Mean flexion angle of the MP joint was significantly smaller in the Surgery group than in the Collagenase group at both 4 and 26 weeks. w, week 